\title{
Factors Affecting Painkillers, Sedatives/Hypnotics, Nicotine, and Unhealthy Alcohol Use Among Gay and Bisexual Men in Taiwan
}

\author{
Dian-Jeng Li ${ }^{1,2} \mathbb{D}$, Shiou-Lan Chen ${ }^{1}$, Yu-Ping Chang ${ }^{3}$ and Cheng-Fang Yen ${ }^{1,4, * \mathbb{C}}$ \\ 1 Graduate Institute of Medicine, College of Medicine, Kaohsiung Medical University, \\ Kaohsiung 80708, Taiwan; u108800004@kmu.edu.tw (D-J.L.); shioulan@kmu.edu.tw (S-L.C.) \\ 2 Department of Addiction Science, Kaohsiung Municipal Kai-Syuan Psychiatric Hospital, \\ Kaohsiung 80276, Taiwan \\ 3 School of Nursing, The State University of New York, University at Buffalo, New York, NY14214-3079, USA; \\ yc73@buffalo.edu \\ 4 Department of Psychiatry, Kaohsiung Medical University Hospital, Kaohsiung 80708, Taiwan \\ * Correspondence: chfaye@cc.kmu.edu.tw; Tel.: +88-6731-21101
}

Received: 7 January 2020; Accepted: 29 January 2020; Published: 29 January 2020

\begin{abstract}
Substance use has become a major health problem globally for sexual minorities. However, few studies have explored multi-dimensional factors associated with smoking, drinking, and prescription drug use. We aimed to investigate the factors affecting painkiller, sedative/hypnotic, nicotine and unhealthy alcohol use among gay and bisexual men in Taiwan. We recruited 500 gay or bisexual men and assessed their experiences of using painkillers, sedatives/hypnotics, nicotine, alcohol and multi-dimensional factors with self-reported questionnaires. Multivariate logistic regression with a forward stepwise model was used to verify the factors associated with substance use. Overall, $9.4 \%, 5.4 \%$, and $13.8 \%$ of the participants reported using painkillers, sedatives/hypnotics, and nicotine, respectively, and 5.6\% reported unhealthy alcohol use. Victims of traditional homophobic bullying in childhood and adolescence were more likely to report nicotine use, sedative/hypnotic use, and unhealthy alcohol use in early adulthood than non-victims. Missing classes or truancy at senior high school was associated with painkiller and sedative/hypnotic use in early adulthood. Traditional homophobic bullying and missing classes or truancy in childhood and adolescence predicted substance use in early adulthood among the gay and bisexual men in this study. Timely preventions and interventions for substance use are crucial for gay and bisexual men, especially for those who experience homophobic bullying and missing classes or truancy.
\end{abstract}

Keywords: sexual minority; prescription drug; smoking; alcohol; homophobic bullying

\section{Introduction}

\subsection{Substance Use in Sexual Minorities}

Substance use has become a major health concern globally for sexual minorities. Problematic substance use increases the mental health burden of sexual minorities. A previous study reported that a third of young men who have sex with men (MSM; $90 \%$ of them identified themselves as gay or bisexual men) who used club drugs (e.g., MDMA or ketamine) had attempted suicide, and that more than half of MSM who regularly use club drugs had a high level of depressive symptoms [1]. Another study reported that polydrug use among gay and bisexual men is predominantly associated with HIV infection and high-risk sexual practices [2]. Although many studies have focused on substance use in 
sexual minorities, few have explored the use of substances other than illicit drugs, such as prescription drugs, in sexual minorities.

\subsection{Use of Painkillers and Sedatives/Hypnotics in Sexual Minorities}

There has recently been increased interest in the misuse of prescription drugs. Painkillers are the most frequently used prescription drug worldwide [3], and painkiller misuse has steadily increased in both the United States and United Kingdom in recent years [4,5]. For sexual minority men, painkillers are also the most prevalent prescription drug, and the risk of misuse cannot be neglected [6]. An epidemiological study regarding prescription drug misuse demonstrated that being bullied at school were associated with painkiller misuse [7]. However, few studies have explored the factors associated with painkiller use in sexual minorities.

Besides painkillers, the use of sedatives/hypnotics such as benzodiazepines is also an important public health concern. A previous study indicated that approximately $12.5 \%$ of US adults used benzodiazepines in 2015 [8]. Benzodiazepines activate GABA/barbiturate receptor sites to produce muscle relaxant effects [7], and they interact with $\alpha \mathrm{GABA}_{\mathrm{A}}$ subunits to enhance sedative and anxiolytic reactions [9]. The weak reinforcing effect of benzodiazepines can lead to their abuse [10]; however, few studies have explored the use of sedatives/hypnotics in sexual minorities. A cohort survey in which $90.2 \%$ of the participants were gay and bisexual men demonstrated a $10.2 \%$ rate of the self-reported use of benzodiazepines in the past 6 months [11]. Another study indicated that frequent transactional sex was associated with benzodiazepine use for MSM of whom 92.1\% were gay and bisexual men [12]. Therefore, the risk factors for sedative/hypnotic use in gay and bisexual men warrant further study.

\subsection{Smoking and Alcohol Use in Sexual Minorities}

Although the prevalence of smoking has gradually fallen in the general population [13], it is still a major concern in sexual minorities. A nationwide study in the US reported that $20.6 \%$ of sexual minority individuals smoked versus $14.9 \%$ of heterosexual individuals [14]. Gay and bisexual men have also been reported to have a 1.4- to 1.6-fold higher rate of smoking than heterosexual men [15]. Several factors associated with patterns of smoking in sexual minorities have been identified. For example, sexual minority women who smoke have been reported to have fewer economic, social and psychological resources than non-smoking sexual minority women [16].

On the other hand, unhealthy alcohol use, defined as drinking to a level above the amount or frequency recommended for the general population [17], often results in higher morbidity and mortality [18], and impacts the mental health of sexual minorities. The National Health Interview Survey in the US reported that gay and bisexual men were more likely to report heavy drinking than heterosexual men [19]. Several studies have explored the impact of social factors on alcohol use specific to gay and bisexual men, and found that they prefer to gather at bars to socialize and meet new partners more than the general population [20], and that this increases their exposure to alcohol-related harm [21]. Moreover, higher income, currently smoking, and greater social discrimination have been associated with a greater likelihood of high-risk drinking in sexual minority men [13]. Further research on the associated risk factors is crucial for clinicians to be able to early identify such issues and initiate appropriate management strategies.

\subsection{Aims of This Study}

We previously comprehensively explored the factors associated with illegal substance use in early adulthood for gay and bisexual men, including school performance, homophobic bullying and social support, and gender-related issues in childhood and adolescence [22]. This research piqued our interest on the use of substances other than heroin, methamphetamines and club drugs. In Taiwan, alcoholic beverages and tobacco are restricted to those who are older than 20 years of age. People can get painkillers and sedatives/hypnotics at a pharmacy with a doctor's prescription. The difference in the availability between "entirely illegal" drugs and "partially restricted" drugs may lead to different 
effects on health outcomes among gay and bisexual men. Furthermore, research on the association between multi-dimensional factors and "partially restricted" substances in gay and bisexual men is still insufficient. Given these gaps in knowledge, the aim of this study was to survey the impacts of multiple factors including traditional/cyber homophobic bullying, disclosure of sexual orientation, perceived family support, and school difficulties during childhood and adolescence on the use of painkillers, sedatives/hypnotics, nicotine and unhealthy alcohol use among sexual minority men in Taiwan.

\section{Materials and Methods}

\subsection{Participants}

The detailed protocol of the current study has been described in our published work [22]. In brief, we recruited gay or bisexual men aged between 20 and 25 years through online and printed advertisements posted on social networking sites and lesbian, gay, bisexual, and transgender (LGBT) clubs. Participants were recruited from August 2015 to July 2017. Individuals who exhibited any cognitive impairment (e.g., intellectual disability or substance intoxication) that could hinder understanding of the purpose of the study or completing the questionnaires were excluded. Before assessment, informed consent was obtained from all participants. In total, 500 males ( 371 gay men and 129 bisexual men) were recruited with a mean age of $22.94 \pm 1.57$ years. All of them provided informed consent prior to their assessment. The study was approved by the Institutional Review Board of Kaohsiung Medical University Hospital (KMUHIRB-F(I)-20150026).

\subsection{Measures}

\subsubsection{Substance Use}

We used the Drug Use Disorders Identification Test-Extended (DUDIT-E) to verify a history of multiple substance use [23]. It had been developed for sequential clinical assessment of drug use. The concurrent validity of D-score is reported to be acceptable, and test-retest reliability is 0.79 , indicating an excellent intraclass correlation [23]. The participants were asked about the frequencies of painkiller, sedative/hypnotic, and tobacco (nicotine) use in the preceding month. Participants who had used any kind of these substances more than once in the preceding month were classified as having specific substance use. A massive epidemiological study indicated that drinking more than 3 to 4 times weekly significantly increased cardiovascular and cancer mortality in men [24]. To identify those with unhealthy alcohol use, we set the cutoff point at "drinking more than twice per week", including the categories of "twice to thrice per week" and "four times per week or above". Hence, this cutoff point can cover those who drink 3 to 4 times weekly. On the other hand, we did not exclude those who are taking painkillers or sedatives/hypnotics in accordance with their medically intended use due to unrecorded information from DUDIT-E.

\subsubsection{Experiences of Traditional and Cyber Homophobic Bullying}

We used six items from the Chinese version of the self-reported School Bullying Experience Questionnaire (C-SBEQ) [25] to assess the experiences of traditional homophobic bullying according to their gender role nonconformity or sexual orientation at school, after-school classes, tutoring schools, and part-time workplaces at elementary (grades 1-6), junior high (grades 7-9), and senior high (grades 10-12) school stages. Multiple types of traditional homophobic bullying were evaluated, including social exclusion, name calling, verbal abuse, physical abuse, forced work, and confiscation of money, school supplies and snacks. The responses for these items were graded on a 4-point Likert scale. A previous study reported that the C-SBEQ had acceptable reliability and validity [25]. The Cronbach $\alpha$ value of the scale for evaluating traditional homophobic bullying was 0.82 in the present study. Based on the results of a previous study [25], we classified the participants who rated 2 or 3 for any item as self-reported victims of traditional homophobic bullying. 
We used three items from the Cyberbullying Experiences Questionnaire [26] to evaluate the participants' experiences of cyberbullying based on their gender role nonconformity or sexual orientation at elementary, junior high, and senior high school stages. These three items addressed the experiences of others posting mean or unpleasant comments; others posting upsetting pictures, photos, or videos; and online rumor-spreading through emails, blogs, social media platforms, and pictures or videos. The Cronbach $\alpha$ value of the scales for evaluating homophobic cyberbullying was 0.81 in this study. Based on the results of a previous study [25], we classified the participants who rated 1 for any item as self-reported victims of homophobic cyberbullying.

\subsubsection{Demographic and Family Characteristics}

We recorded information of the participants' age, educational level, parental marriage status, and parental education levels. The participants were classified into those with a high education level (college or above) and those with a low education level (high school or below). They were also classified into those with high parental education levels (both parents completed 9 years of compulsory fundamental education) and those with low parental education levels (any parent did not complete 9 years of compulsory fundamental education).

\subsubsection{Sexual Orientation Characteristics}

The participants' sexual orientation (homosexual or bisexual) and time of disclosure of sexual orientation (elementary school, junior high school, senior high school, and college) was recorded.

\subsubsection{Social Support}

The Chinese version of the 5-item self-administered Family Adaptation, Partnership, Growth, Affection, Resolve (APGAR) Index was used to measure the participants' satisfaction with family support during their childhood and adolescence $[27,28]$. Each item was rated on a 4-point Likert scale, with scores ranging from 0 (never) to 3 (always). In addition, we used the Peer APGAR Index, which is adapted from the Family APGAR Index, to measure the participants' satisfaction with peer support during childhood and adolescence [29]. Higher total scores on the Family and Peer APGAR Indices indicated higher levels of family and peer support, respectively. The Cronbach $\alpha$ values for the Family and Peer APGAR Indices in this study were 0.86 and 0.87 , respectively.

\subsubsection{School Characteristics}

The participants were asked to rate their subjective satisfaction with their academic performance at elementary, junior high, and senior high schools using a 4-point Likert scale, ranging from 0 (very satisfied) to 3 (not satisfied at all). The participants who responded 2 or 3 were identified as being dissatisfied with their academic performance. The tendency to miss classes or be truant at elementary, junior high, and senior high schools was evaluated using a 4-point Likert scale, ranging from 0 (never) to 3 (very frequent). The participants who did not answer 0 on any item were classified as having a tendency to miss classes or be truant.

\subsection{Procedure}

This cross-sectional study used a paper-and-pencil questionnaire. Research assistants explained the procedures and methods for completing the questionnaires to the participants individually. The participants could ask any question if they had difficulty in completing the questionnaires, and the research assistants resolved any problems.

\subsection{Statistical Analysis}

Initially, the demographic, sexual orientation, family, school and social support characteristics were summarized. Univariate logistic regression was used to examine potential factors associated with painkillers, 
sedatives/hypnotics, nicotine, and unhealthy alcohol use. All significant variables identified in the first step were then entered into a forward stepwise logistic regression model to determine the best predictors. Prevalence ratios (PRs) and 95\% confidence intervals (CIs) were used to present the statistical significance. To estimate the correlation or dependency between variables, the Pearson correlation was used to determine the linear relationships between bullying (traditional or cyber) and missing classes/truancy or satisfaction with academic performance. All tests were 2-tailed, and statistical significance was set at $p<0.05$. All data were processed using SPSS version 23.0 for Windows (SPSS Inc., Chicago, IL, USA).

\section{Results}

\subsection{Patient Variables}

Table 1 presents the distributions and frequencies of substances use in the 500 participants. In total, $9.4 \%, 5.4 \%$, and $13.8 \%$ of the participants reported using painkillers, sedatives/hypnotics, and smoking at least once per month but less than twice per month, respectively, and 5.6\% reported using alcohol at least twice per week (unhealthy alcohol use).

Table 1. Distribution of and frequency of painkillers, sedative/hypnotic drugs, nicotine, and unhealthy alcohol use among 500 participants.

\begin{tabular}{ccccccccc}
\hline Type of Substance & \multicolumn{2}{c}{ Painkillers } & \multicolumn{2}{c}{ Sedative/Hypnotics } & \multicolumn{2}{c}{ Nicotine } & \multicolumn{2}{c}{ Unhealthy Alcohol Use } \\
\hline Distribution & $n$ & $\%$ & $n$ & $\%$ & $n$ & $\%$ & $n$ & $\%$ \\
\hline Never used in recent one year & 414 & 82.8 & 456 & 91.2 & 417 & 83.4 & 178 & 35.6 \\
Ever used but less than once per month & 39 & 7.8 & 17 & 3.4 & 14 & 2.8 & 94 & 18.8 \\
Once per month but less than twice per month & 21 & 4.2 & 5 & 1.0 & 9 & 1.8 & 107 & 21.4 \\
Twice to four times per month & 18 & 3.6 & 9 & 1.8 & 7 & 1.4 & 93 & 18.6 \\
Twice to thrice per week & 5 & 1.0 & 4 & 0.8 & 5 & 1.0 & 15 & 3.0 \\
Four times per week or above & 3 & 0.6 & 9 & 1.8 & 48 & 9.6 & 13 & 2.6 \\
\hline
\end{tabular}

Data on the demographics, sexual orientation, traditional and cyber homophobic bullying, family and school characteristics, and social support of the participants are listed in Tables 2-5.

Table 2. Associations of demographic factors, sexual orientation and victimization of homophobic bullying with painkillers and sedatives/hypnotics use examined by univariate logistic regression $(n=500)$.

\begin{tabular}{|c|c|c|c|c|c|c|c|c|}
\hline & \multirow[b]{2}{*}{ Mean } & \multirow[b]{2}{*}{ SD } & \multicolumn{3}{|c|}{ Painkillers } & \multicolumn{3}{|c|}{ Sedatives/Hypnotics } \\
\hline & & & PR & $95 \%$ CI & $p$ & PR & $95 \%$ CI & $p$ \\
\hline \multirow[t]{2}{*}{ Age (years) } & 22.94 & 1.57 & 1.07 & $0.88-1.29$ & 0.525 & 1.01 & $0.78-1.29$ & 0.971 \\
\hline & $n$ & $\%$ & OR & $95.0 \%$ of $\mathrm{CI}$ & $p$ & OR & $95 \% \mathrm{CI}$ & $p$ \\
\hline \multicolumn{9}{|l|}{ Education level } \\
\hline High (college or above) & 450 & 90 & & & & & & \\
\hline Low (high school or below) & 50 & 10 & 1.36 & $0.55-3.38$ & 0.508 & 0.71 & $0.16-3.08$ & 0.646 \\
\hline \multicolumn{9}{|l|}{ Sexual orientation identity } \\
\hline Gay & 371 & 74.2 & & & & & & \\
\hline Bisexual & 129 & 25.8 & 0.87 & $0.43-1.76$ & 0.694 & 1.01 & $0.42-2.44$ & 0.988 \\
\hline \multirow{2}{*}{\multicolumn{9}{|c|}{$\begin{array}{c}\text { Victims of homophobic traditional } \\
\text { bullying }\end{array}$}} \\
\hline & & & & & & & & \\
\hline Yes & 190 & 38 & 1.80 & $0.99-3.30$ & 0.055 & 3.50 & $1.54-7.96$ & 0.003 \\
\hline \multicolumn{9}{|l|}{$\begin{array}{l}\text { Victims of homophobic } \\
\text { cyberbullying }\end{array}$} \\
\hline No & 299 & 59.8 & & & & & & \\
\hline Yes & 201 & 40.2 & 1.97 & $1.07-3.60$ & 0.029 & 2.67 & $1.20-5.96$ & 0.016 \\
\hline \multicolumn{9}{|l|}{ Time to disclose sexual orientation } \\
\hline \multicolumn{9}{|l|}{ At elementary school } \\
\hline No & 476 & 95.2 & & & & & & \\
\hline Yes & 24 & 4.8 & 1.40 & $0.40-4.90$ & 0.595 & 0.75 & $0.10-5.79$ & 0.785 \\
\hline \multicolumn{9}{|l|}{ At junior high school } \\
\hline No & 365 & 73 & & & & & & \\
\hline Yes & 135 & 27 & 2.18 & $1.18-4.03$ & 0.013 & 2.28 & $1.04-5.00$ & 0.004 \\
\hline \multicolumn{9}{|l|}{ At senior high school } \\
\hline No & 215 & 43 & & & & & & \\
\hline Yes & 285 & 57 & 2.37 & $1.20-4.68$ & 0.013 & 0.94 & $0.43-2.05$ & 0.940 \\
\hline \multicolumn{9}{|l|}{ At college or above } \\
\hline No & 53 & 10.6 & & & & & & \\
\hline Yes & 447 & 89.4 & 5.97 & $0.81-44.17$ & 0.08 & 1.51 & $0.35-6.57$ & 0.582 \\
\hline
\end{tabular}

$\mathrm{CI}=$ Confidence interval; $\mathrm{PR}=$ Prevalence ratio, ratio of odds of illegal substance use vs. non-use among participants; $\mathrm{SD}=$ Standard deviation; Bolds: statistical significance $(p<0.05)$. 
Table 3. Family, peer and school factors related to painkillers and sedatives/hypnotics use examined by univariate logistic regression $(n=500)$.

\begin{tabular}{|c|c|c|c|c|c|c|c|c|}
\hline & \multirow[b]{2}{*}{ Mean } & \multirow[b]{2}{*}{ SD } & \multicolumn{4}{|c|}{ Painkillers } & \multicolumn{2}{|c|}{ Sedatives/Hypnotics } \\
\hline & & & PR & $95 \% \mathrm{CI}$ & $p$ & PR & $95 \% \mathrm{CI}$ & $p$ \\
\hline Perceived family support on the APGAR & 8.49 & 3.83 & 0.93 & $0.86-1.01$ & 0.08 & 0.81 & $0.73-0.90$ & $<0.001$ \\
\hline \multirow[t]{2}{*}{ Perceived peer support on the APGAR } & 11.42 & 2.89 & 0.98 & $0.93-1.16$ & 0.516 & 0.91 & $0.81-1.02$ & 0.112 \\
\hline & $n$ & $\%$ & OR & $95.0 \% \mathrm{CI}$ & $p$ & OR & $95 \% \mathrm{CI}$ & $p$ \\
\hline \multicolumn{9}{|l|}{ Parental marital status } \\
\hline Married and living together & 328 & 65.6 & - & _ & _ & & & \\
\hline Separated or divorced & 136 & 27.2 & 1.44 & $0.74-2.81$ & 0.286 & 2.17 & $0.98-4.82$ & 0.057 \\
\hline Widowed & 36 & 7.2 & 2.32 & $0.89-6.09$ & 0.086 & 0.64 & $0.08-5.02$ & 0.672 \\
\hline \multicolumn{9}{|l|}{ Paternal education level } \\
\hline High (senior high school or above) & 385 & 77 & & & & & & \\
\hline Low (junior high school or below) & 115 & 23 & 0.66 & $0.30-1.46$ & 0.309 & 0.40 & $0.12-1.36$ & 0.144 \\
\hline \multicolumn{4}{|l|}{ Maternal education level } & & & & & \\
\hline Low (junior high school or below) & 112 & 22.4 & 1.07 & $0.52-2.17$ & 0.862 & 0.42 & $0.12-1.41$ & 0.160 \\
\hline \multicolumn{9}{|l|}{$\begin{array}{l}\text { Satisfaction with academic performance } \\
\text { In elementary school }\end{array}$} \\
\hline High & 401 & 80.2 & & & & & & \\
\hline Low & 99 & 19.8 & 1.44 & $0.72-2.89$ & 0.302 & 1.77 & $0.75-4.17$ & 0.193 \\
\hline \multicolumn{9}{|l|}{ In junior high school } \\
\hline High & 336 & 67.2 & & & & & & \\
\hline Low & 164 & 32.8 & 1.44 & $0.78-2.67$ & 0.244 & 1.69 & $0.77-3.70$ & 0.189 \\
\hline \multicolumn{9}{|l|}{ In senior high school } \\
\hline High & 310 & 62.0 & & & & & & \\
\hline Low & 190 & 38.0 & 0.92 & $0.49-1.71$ & 0.917 & 2.50 & $1.13-5.51$ & 0.023 \\
\hline \multicolumn{9}{|l|}{ Missing classes or truancy } \\
\hline \multicolumn{9}{|l|}{ In elementary school } \\
\hline No & 456 & 91.2 & & & & & & \\
\hline Yes & 44 & 8.8 & 1.60 & $0.64-4.00$ & 0.317 & 2.53 & $0.91-7.05$ & 0.076 \\
\hline \multicolumn{9}{|l|}{ In junior high school } \\
\hline No & 414 & 82.8 & & & & & & \\
\hline Yes & 86 & 17.2 & 1.76 & $0.87-3.54$ & 0.116 & 3.07 & $1.36-6.97$ & 0.007 \\
\hline \multicolumn{9}{|l|}{ In senior high school } \\
\hline No & 359 & 71.8 & & & & & & \\
\hline Yes & 141 & 28.2 & 2.47 & $1.34-4.55$ & 0.004 & 4.79 & $2.13-10.73$ & $<0.001$ \\
\hline
\end{tabular}

$\overline{\mathrm{CI}}=$ Confidence interval; $\mathrm{PR}=$ Prevalence ratio; $\mathrm{SD}=$ Standard deviation; Bolds: statistical significance $(p<0.05)$.

Table 4. Associations of demographic factors, sexual orientation and victimization of homophobic bullying with nicotine and unhealthy alcohol use examined by univariate logistic regression $(n=500)$.

\begin{tabular}{|c|c|c|c|c|c|c|c|c|}
\hline & \multirow[b]{2}{*}{ Mean } & \multirow[b]{2}{*}{ SD } & \multicolumn{4}{|c|}{ Nicotine } & \multicolumn{2}{|c|}{ Alcohol } \\
\hline & & & PR & $95 \%$ CI & $p$ & PR & $95 \%$ CI & $p$ \\
\hline \multirow[t]{2}{*}{ Age (years) } & 22.94 & 1.57 & 0.93 & $0.79-1.09$ & 0.374 & 0.84 & $0.66-1.07$ & 0.156 \\
\hline & $n$ & $\%$ & OR & $95.0 \%$ of $\mathrm{CI}$ & $p$ & OR & $95 \% \mathrm{CI}$ & $\mathrm{p}$ \\
\hline \multicolumn{9}{|l|}{ Education level } \\
\hline High (college or above) & 450 & 90 & & & & & & \\
\hline Low (high school or below) & 50 & 10 & 3.53 & $1.82-6.82$ & $<0.001$ & 2.06 & $0.75-5.69$ & 0.162 \\
\hline \multicolumn{9}{|l|}{ Sexual orientation identity } \\
\hline Gay & 371 & 74.2 & & & & & & \\
\hline Bisexual & 129 & 25.8 & 1.66 & $0.96-2.85$ & 0.068 & 1.39 & $0.61-3.15$ & 0.432 \\
\hline \multicolumn{9}{|l|}{$\begin{array}{l}\text { Victims of homophobic } \\
\text { traditional bullying }\end{array}$} \\
\hline No & 310 & 62 & & & & & & \\
\hline Yes & 190 & 38 & 2.25 & $1.35-3.76$ & 0.002 & 3.14 & $1.42-6.96$ & 0.005 \\
\hline \multicolumn{9}{|l|}{$\begin{array}{l}\text { Victims of homophobic } \\
\text { cyberbullying }\end{array}$} \\
\hline No & 299 & 59.8 & & & & & & \\
\hline Yes & 201 & 40.2 & 2.31 & $1.38-3.88$ & 0.001 & 2.84 & $1.28-6.29$ & 0.01 \\
\hline \multicolumn{9}{|l|}{$\begin{array}{l}\text { Time to disclose sexual } \\
\text { orientation }\end{array}$} \\
\hline \multicolumn{9}{|l|}{ At elementary school } \\
\hline No & 476 & 95.2 & & & & & & \\
\hline Yes & 24 & 4.8 & 1.27 & $0.42-3.82$ & 0.677 & 1.57 & $0.35-7.06$ & 0.554 \\
\hline \multicolumn{9}{|l|}{ At junior high school } \\
\hline No & 365 & 73 & & & & & & \\
\hline Yes & 135 & 27 & 0.95 & $0.53-1.69$ & 0.854 & 1.54 & $0.69-3.43$ & 0.288 \\
\hline \multicolumn{9}{|l|}{ At senior high school } \\
\hline No & 215 & 43 & & & & & & \\
\hline Yes & 285 & 57 & 0.98 & $0.59-1.63$ & 0.931 & 1.38 & $0.63-3.06$ & 0.425 \\
\hline \multicolumn{9}{|l|}{ At college or above } \\
\hline No & 53 & 10.6 & & & & & & \\
\hline Yes & 447 & 89.4 & 0.76 & $0.35-1.63$ & 0.479 & 0.52 & $0.19-1.43$ & 0.206 \\
\hline
\end{tabular}

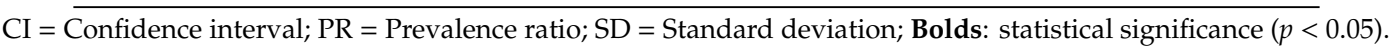


Table 5. Family, peer and school factors associated with nicotine and unhealthy alcohol use examined by univariate logistic regression $(n=500)$.

\begin{tabular}{|c|c|c|c|c|c|c|c|c|}
\hline & & & & Nicotine & & & Alcohol & \\
\hline & Mean & SD & PR & $95 \% \mathrm{CI}$ & $p$ & PR & $95 \% \mathrm{CI}$ & $p$ \\
\hline $\begin{array}{c}\text { Perceived family support on the } \\
\text { APGAR }\end{array}$ & 8.49 & 3.83 & 0.90 & $0.84-0.96$ & 0.002 & 0.96 & $0.87-1.06$ & 0.457 \\
\hline \multirow[t]{2}{*}{$\begin{array}{l}\text { Perceived peer support on the } \\
\text { APGAR }\end{array}$} & 11.42 & 2.89 & 1.01 & $0.93-1.11$ & 0.753 & 0.97 & $0.85-1.10$ & 0.602 \\
\hline & $n$ & $\%$ & OR & $95.0 \% \mathrm{CI}$ & $p$ & OR & $95 \% \mathrm{CI}$ & $p$ \\
\hline \multicolumn{9}{|l|}{ Parental marital status } \\
\hline Married and living together & 328 & 65.6 & - & - & - & & & \\
\hline Separated or divorced & 136 & 27.2 & 1.28 & $0.72-2.26$ & 0.397 & 0.962 & $0.94-3.49$ & 0.077 \\
\hline Widowed & 36 & 7.2 & 1.69 & $0.70-4.11$ & 0.247 & $<0.001$ & $<0.001-<0.001$ & 0.998 \\
\hline \multicolumn{9}{|l|}{ Paternal education level } \\
\hline High (senior high school or above) & 385 & 77 & & & & & & \\
\hline \multicolumn{8}{|l|}{ Maternal education level } & 0.057 \\
\hline High (senior high school or above) & 388 & 77.6 & & & & & & \\
\hline Low (junior high school or below) & 112 & 22.4 & 0.62 & $0.31-1.23$ & 0.169 & 0.25 & $0.06-1.08$ & 0.064 \\
\hline \multicolumn{9}{|l|}{$\begin{array}{l}\text { Satisfaction with academic } \\
\text { performance }\end{array}$} \\
\hline \multicolumn{9}{|l|}{ In elementary school } \\
\hline High & 401 & 80.2 & & & & & & \\
\hline Low & 99 & 19.8 & 1.04 & $0.55-1.95$ & 0.912 & 0.87 & $0.32-2.36$ & 0.791 \\
\hline \multicolumn{9}{|l|}{ In junior high school } \\
\hline High & 336 & 67.2 & & & & & & \\
\hline Low & 164 & 32.8 & 1.19 & $0.70-2.03$ & 0.513 & 0.97 & $0.43-2.19$ & 0.939 \\
\hline \multicolumn{9}{|l|}{ In senior high school } \\
\hline High & 310 & 62.0 & & & & & & \\
\hline Low & 190 & 38.0 & 1.06 & $0.63-1.78$ & 0.835 & 0.53 & $0.22-1.26$ & 0.151 \\
\hline \multicolumn{9}{|l|}{ Missing classes or truancy } \\
\hline No & 456 & 91.2 & & & & & & \\
\hline Yes & 44 & 8.8 & 0.60 & $0.21-1.74$ & 0.348 & 0.79 & $0.18-3.44$ & 0.751 \\
\hline \multicolumn{9}{|l|}{ In junior high school } \\
\hline No & 414 & 82.8 & & & & & & \\
\hline Yes & 86 & 17.2 & 1.72 & $0.94-3.14$ & 0.081 & 1.66 & $0.68-4.03$ & 0.265 \\
\hline \multicolumn{9}{|l|}{ In senior high school } \\
\hline No & 359 & 71.8 & & & & & & \\
\hline Yes & 141 & 28.2 & 2.94 & $1.75-4.95$ & $<0.001$ & 1.99 & $0.92-4.33$ & 0.081 \\
\hline
\end{tabular}

\subsection{Predictors of Painkillers, Sedatives/Hypnotics, Nicotine, and Unhealthy Alcohol Use}

The results of the univariate logistic regression analysis were as follows. Being victims of homophobic cyberbullying, disclosure of sexual orientation at junior high school and at senior high school, and missing classes or truancy at senior high school were significantly associated with the use of painkillers. Being victims of traditional homophobic bullying, being victims of homophobic cyberbullying, disclosure of sexual orientation at junior high school, lower Family APGAR Index score, lower satisfaction with academic performance at senior high school, missing classes or truancy at junior high school and senior high school were significantly associated with the use of sedatives/hypnotics. Lower educational level, being victims of traditional homophobic bullying, being victims of homophobic cyberbullying, lower Family APGAR Index score, higher paternal educational level (higher as indicator: $\mathrm{PR}=2.17, p=0.038$; lower as indicator: $\mathrm{PR}=0.46, p=0.038$ ), and missing classes or truancy at senior high school were significantly associated with nicotine use. Being victims of traditional homophobic bullying and being victims of homophobic cyberbullying were significantly associated with unhealthy alcohol use (Tables 2-5).

The results of the forward stepwise logistic regression analysis were as follows (Table 6). Disclosure of sexual orientation at senior high school and missing classes or truancy at senior high school were significantly associated with the use of painkillers. Being victims of traditional homophobic bullying, lower Family APGAR Index score, and missing classes or truancy at senior high school were significantly associated with the use of sedatives/hypnotics. Being victims of traditional homophobic bullying, being victims of homophobic cyberbullying, lower educational level, higher paternal educational level (higher as indicator: $\mathrm{PR}=2.49, p=0.019$; lower as indicator: $\mathrm{PR}=0.40 ; p=0.019$ ), and missing 
classes or truancy at senior high school were significantly associated with nicotine use. Being victims of traditional homophobic bullying was significantly associated with unhealthy alcohol use.

Table 6. Predictors of painkillers, sedatives/hypnotics, nicotine, and unhealthy alcohol use examined using forward stepwise logistical regression.

\begin{tabular}{cccc}
\hline Predictors of Painkillers Use & PR & 95\% CI & $p$ \\
\hline Disclosure of sexual orientation at senior high school & 2.35 & $1.18-4.67$ & $\mathbf{0 . 0 1 5}$ \\
Missing classes or truancy in senior high school & 2.45 & $1.33-4.53$ & $\mathbf{0 . 0 0 4}$ \\
\hline Predictors of sedatives/hypnotics use & OR & $95 \%$ CI & $p$ \\
\hline Victims of homophobic traditional bullying & 2.65 & $1.12-6.25$ & $\mathbf{0 . 0 2 7}$ \\
Perceived family support on the APGAR & 0.85 & $0.76-0.95$ & $\mathbf{0 . 0 0 3}$ \\
Missing classes or truancy in senior high school & 4.02 & $1.75-9.24$ & $\mathbf{0 . 0 0 1}$ \\
\hline Predictors of nicotine use & OR & $95 \%$ CI & $p$ \\
\hline Victims of homophobic traditional bullying & 1.92 & $1.10-3.34$ & $\mathbf{0 . 0 2 2}$ \\
Victims of homophobic cyberbullying & 1.78 & $1.02-3.10$ & $\mathbf{0 . 0 4 2}$ \\
Missing classes or truancy in senior high school & 2.46 & $1.43-4.25$ & $\mathbf{0 . 0 0 1}$ \\
Paternal education level & 0.40 & $0.19-0.86$ & $\mathbf{0 . 0 1 9}$ \\
Education level & 2.73 & $1.34-5.55$ & $\mathbf{0 . 0 0 6}$ \\
\hline Predictors of unhealthy alcohol use & OR & $95 \%$ CI & $p$ \\
\hline Victims of homophobic traditional bullying & 3.14 & $1.42-6.96$ & $\mathbf{0 . 0 0 5}$ \\
\hline
\end{tabular}

PR = Prevalence ratio; Bolds: statistical significance $(p<0.05)$.

The correlations between bullying and school factors (missing classes/truancy and satisfaction with academic performance) were estimated. The correlations with cyberbullying are as follows: truancy in elementary school $(\mathrm{r}=0.03 ; p=0.458)$, truancy in junior high school $(\mathrm{r}=0.19 ; p<0.001)$, truancy in senior high school $(\mathrm{r}=0.09 ; p=0.037)$, satisfaction in elementary school $(\mathrm{r}=0.05 ; p=0.235)$, satisfaction in junior high school $(\mathrm{r}=0.16 ; p<0.001)$, satisfaction in senior high school $(\mathrm{r}=0.07 ; p=$ 0.106). On the other hand, the correlation with traditional bullying are as follows: truancy in elementary school $(\mathrm{r}=0.03 ; p=0.459)$, truancy in junior high school $(\mathrm{r}=0.11 ; p=0.012)$, truancy in senior high school $(\mathrm{r}=0.05 ; p=0.268)$, satisfaction in elementary school $(\mathrm{r}=0.12 ; p=0.008)$, satisfaction in junior high school $(\mathrm{r}=0.17 ; p<0.001)$, satisfaction in senior high school $(\mathrm{r}=0.03 ; p=0.472)$. Among them, the effect sizes of significant correlations are low regarding the cutoff values of Pearson correlation.

\section{Discussion}

\subsection{The Main Findings of the Present Study}

We found that painkiller use was associated with the disclosure of sexual orientation at junior high school and missing classes or truancy at senior high school among the Taiwanese gay and bisexual men in this study. The use of sedatives/hypnotics was associated with traditional homophobic bullying in childhood and adolescence, lower family support, and missing classes or truancy at senior high school. Nicotine use was associated with traditional/cyber homophobic bullying in childhood and adolescence, higher paternal educational level, lower educational level, and missing classes or truancy at senior high school. Unhealthy alcohol use was associated with traditional homophobic bullying in childhood and adolescence.

\subsection{Characteristics of Substance Use in Sexual Minorities}

In this study, $9.4 \%$ of the recruited gay and bisexual men took painkillers at least once per month, which is different to previous studies. An epidemiological investigation in the elderly found that analgesics were the most common over-the-counter medication in 2003-2004, with a prevalence of 
$8.8 \%$ [3]. A study on MSM of whom $98.5 \%$ were gay and bisexual reported that $11.3 \%$ of the participants had taken painkillers in the past 3 months [30], which is comparable to our results. However, they reported a higher 3-month prevalence of sedatives (8.3\%) and sleep aids (16.3\%) than in our study (5.4\% at least once per month). On the other hand, the smoking rate in our study ( $13.8 \%$ at least once per month) was lower than in a prospective study reported by Fallin et al. (25.9\% for gay and $33.7 \%$ for bisexual men) [31]. The differences between the results may be due to differences in measurements. We chose "at least once per month" as the cutoff point to identify those who took painkillers continuously, and also for the use of nicotine and sedatives/hypnotics. Moreover, no previous study has investigated unhealthy alcohol use (more than 14 drinks per week) in sexual minorities.

\subsection{Traditional/Cyber Homophobic Bullying and Disclosure of Sexual Orientation Associated with Substance Use}

Traditional homophobic bullying was a major risk factor for sedatives/hypnotics, nicotine, and unhealthy alcohol use in this study. We also found an association between traditional homophobic bullying and illegal substance use in our previous work [22]. Homophobic bullying has been reported to be a serious risk factor for alcohol use among sexual minority youth [32]. In another longitudinal study, Tucker et al. did not find an association between homophobic name-calling and alcohol use within sexual minority students, although the psychological stress was significantly increased [33]. This difference may be due to the 1-year observation period in Tucker et al.'s study, and because we investigated the impact of homophobic bullying in childhood and adolescence, which may further explain the effect of alcohol use in early adulthood.

In our previous work, we found that victims of traditional homophobic bullying had more severe physical pain in adulthood [29]. Therefore, it is reasonable that the use of painkillers is associated with traditional homophobic bullying. In addition, perceived discrimination was associated with an increased risk of current smoking in a multiethnic population-based study [34], and we found a similar association in gay and bisexual men. Furthermore, we identified the impact of traditional homophobic bullying on sedative/hypnotic use, which has rarely been reported. Although we did not find that homophobic bullying was a powerful predictor for painkiller use in forward stepwise logistic regression, we found that disclosure of sexual orientation at senior high school remained a major risk factor. Disclosure of one's sexual orientation has been reported to increase psychosocial stress in sexual minorities [35]. It is possible that individuals who disclose their sexual orientation may suffer from homophobic victimization and, consequently, substance use.

Besides traditional homophobic bullying, we also found that homophobic cyberbullying in childhood and adolescence was associated with smoking in early adulthood. Although both traditional bullying and cyberbullying were risk factors, cyberbullying has been reported to have different characteristics from traditional bullying, such as anonymity and individualistic activities [36]. Cyberbullying has also been associated with smoking in Italian adolescents [37]. We further confirmed this association among the gay and bisexual men in this study. Previous studies have used "Minority Stress Theory" [38] to verify the association between homophobic victimization and substance use in sexual minorities [39]. Investigations of the risk factors of substance use in sexual minorities may benefit the development of prevention and intervention programs. Our results also support the need for preventions and interventions for traditional/cyber homophobic bullying in gay and bisexual men.

\subsection{School Factors and Education Level Associated with Substance Use}

We found that missing classes or truancy at senior high school was significantly associated with multiple substance use in early adulthood, including painkillers, sedatives/hypnotics, and nicotine. A previous study also reported that truancy was associated with substance use in children and adolescents [40]. However, no previous study has explored this association among sexual minorities. In our previous work, we found an association between illegal substance use and truancy among gay and bisexual men [22], and the current study extends these findings to painkillers, sedatives/hypnotics, 
and nicotine. On the other hand, in the present study, we also found that a lower educational level was significantly associated with nicotine use. A lower educational level has been reported to be related with smoking in sexual minority women [41]. Another study reported that LGBT individuals with a higher education level had lower odds of smoking [42]. Our study showed similar results for nicotine use among gay and bisexual men.

\subsection{Family Factors Associated with Substance Use}

The use of sedatives/hypnotics and nicotine was associated with a lower Family APGAR Index score in this study, which means poorer family support. Poor family management among adolescents has also been associated with increased alcohol and other drug use [43]. In addition, a previous study indicated that family rejection predicted substance use and related problems in sexual minorities [44]. A more supportive LGBT environment in schools and communities has been reported to result in less substance use in sexual minority adolescents [45]. We also identified the importance of family support in childhood and adolescence to prevent substance use in early adulthood; however, we did not find a significant association between peer support during childhood and adolescence and substance use in early adulthood.

We also found that a higher paternal educational level was significantly associated with nicotine and potentially related with unhealthy alcohol use, which has rarely been reported previously. Greater paternal demandingness has been significantly associated with binge drinking in Argentinean youth [46]. To date, no published study has investigated this association. We hypothesize that fathers with a higher educational level may have higher expectations of their children, which may then lead to increased stress and, consequently, substance use.

\subsection{Limitations}

We presented the comprehensive results of the associations between multi-dimensional factors and substance use; however, several limitations of the current study should be noted. First, we did not record the doses of the substances used, so we could not precisely estimate the harm done to the participants. Second, the sources of painkillers and sedatives/hypnotics were also unrecorded, so that it was difficult to identify whether or not they had prescriptions for the drugs. This may have led to underestimation of the potential abuse of prescription drugs. Third, given that we did not ask the age at initial substance use, we could not identify temporal relationships between substance use and related factors with age. Fourth, this self-reported study obtained data on homophobic bullying of the participants, school factors and family and peer support retrospectively. Hence, recall bias may have confounded the results. Fifth, the mean age of recruited subjects was relatively young (20 25 years old), which limited to the generalizability to other birth cohorts. Finally, we recruited only gay and bisexual men, so our findings may not be applicable to lesbian or other sexual minorities, and further studies are warranted to generalize out result to the broader LGBT community in Taiwan.

\section{Conclusions}

In this study, we found that multi-dimensional factors were associated with substance use, including painkillers, nicotine, sedatives/hypnotics, and unhealthy alcohol use. To be specific, we provided new information that homophobic bullying and school-related factors in childhood and adolescence affected substance use in early adulthood. Our results revealed that mental health professionals should regularly assess histories of homophobic bullying and school-related factors when assessing gay and bisexual men with substance use. Gay-affirmative cognitive behavioral therapy has been shown to be beneficial for victims of homophobic bullying in childhood and adolescence [47], and timely treatment for psychological trauma from bullying may prevent substance use due to maladaptive behaviors. We suggest that gender-friendly/neutral policy in school is beneficial to prevent substance use, and we provide useful information for the development of prevention and early intervention programs for substance use in gay and bisexual men. 
Author Contributions: Conceptualization, C-F.Y. and D-J.L.; Methodology, D-J.L., S-L.C. and Y-P.C.; Software, D-J.L. and S-L.C.; Validation, D-J.L., S-L.C. and Y-P.C.; Formal Analysis, D-J.L., S-L.C. and Y-P.C..; Investigation, C-F.Y. and D-J.L.; Resources, C-F.Y.; Data Curation, C-F.Y..; Writing-Original Draft Preparation, D-J.L..; Writing-Review \& Editing, D-J.L. and C-F.Y.; Visualization, D-J.L.; Supervision, C-F.Y.; Project Administration, C-F.Y.; Funding Acquisition, C-F.Y. All authors have read and agreed to the published version of the manuscript.

Funding: The present study was supported by the Ministry of Science and Technology, Taiwan, R.O.C. (Grant no. MOST 104-2314-B-037-024-MY3) and the Kaohsiung Medical University Hospital (Grant nos. KMUH104-4R60, KMUH105-5R59 and KMUH106-6R67). The funders had no role in the design of the study; in the collection, analyses, or interpretation of data; in the writing of the manuscript, or in the decision to publish the results.

Conflicts of Interest: The authors declare no conflict of interest.

\section{References}

1. Clatts, M.C.; Goldsamt, L.A.; Yi, H. Club drug use among young men who have sex with men in NYC: A preliminary epidemiological profile. Subst. Use. Misuse 2005, 40, 1317-1330. [CrossRef] [PubMed]

2. Stall, R.; Mills, T.C.; Williamson, J.; Hart, T.; Greenwood, G.; Paul, J.; Pollack, L.; Binson, D.; Osmond, D.; Catania, J.A. Association of co-occurring psychosocial health problems and increased vulnerability to HIV/AIDS among urban men who have sex with men. Am. J. Public Health 2003, 93, 939-942. [CrossRef] [PubMed]

3. Goh, L.Y.; Vitry, A.I.; Semple, S.J.; Esterman, A.; Luszcz, M.A. Self-medication with over-the-counter drugs and complementary medications in South Australia's elderly population. Bmc. Complement. Altern. Med. 2009, 9, 42. [CrossRef] [PubMed]

4. Compton, W.M.; Volkow, N.D. Abuse of prescription drugs and the risk of addiction. Drug Alcohol Depend. 2006, 83 Suppl. 1, S4-S7. [CrossRef] [PubMed]

5. Lessenger, J.E.; Feinberg, S.D. Abuse of prescription and over-the-counter medications. J. Am. Board Fam. Med. 2008, 21, 45-54. [CrossRef] [PubMed]

6. Li, D.H.; Mustanski, B. Prevalence and Correlates of Prescription Drug Misuse Among a Racially Diverse Sample of Young Sexual Minority Men. Lgbt Health 2018, 5, 95-104. [CrossRef]

7. Baiden, P.; Tadeo, S.K. Examining the association between bullying victimization and prescription drug misuse among adolescents in the United States. J. Affect. Disord 2019, 259, 317-324. [CrossRef]

8. Blanco, C.; Han, B.; Jones, C.M.; Johnson, K.; Compton, W.M. Prevalence and Correlates of Benzodiazepine Use, Misuse, and Use Disorders Among Adults in the United States. J. Clin. Psychiatry 2018, 79. [CrossRef]

9. McKernan, R.M.; Rosahl, T.W.; Reynolds, D.S.; Sur, C.; Wafford, K.A.; Atack, J.R.; Farrar, S.; Myers, J.; Cook, G.; Ferris, P.; et al. Sedative but not anxiolytic properties of benzodiazepines are mediated by the GABA(A) receptor alpha1 subtype. Nat. Neurosci. 2000, 3, 587-592. [CrossRef]

10. Ator, N.A. Contributions of GABAA receptor subtype selectivity to abuse liability and dependence potential of pharmacological treatments for anxiety and sleep disorders. Cns. Spectr. 2005, 10, 31-39. [CrossRef]

11. Li, D.H.; Janulis, P.; Mustanski, B. Predictors of correspondence between self-reported substance use and urinalysis screening among a racially diverse cohort of young men who have sex with men and transgender women. Addict. Behav. 2019, 88, 6-14. [CrossRef] [PubMed]

12. Berg, R.C.; Weatherburn, P.; Marcus, U.; Schmidt, A.J. Links between transactional sex and HIV/STI-risk and substance use among a large sample of European men who have sex with men. Bmc. Infect. Dis. 2019, 19, 686. [CrossRef] [PubMed]

13. Agaku, I.T.; King, B.A.; Dube, S.R.; Centers for Disease Control and Prevention. Current cigarette smoking among adults - United States, 2005-2012. Mmwr. Morb. Mortal Wkly. Rep. 2014, 63, 29-34. [PubMed]

14. Jamal, A.; King, B.A.; Neff, L.J.; Whitmill, J.; Babb, S.D.; Graffunder, C.M. Current Cigarette Smoking Among Adults - United States, 2005-2015. Mmwr. Morb. Mortal Wkly. Rep. 2016, 65, 1205-1211. [CrossRef]

15. Gruskin, E.P.; Greenwood, G.L.; Matevia, M.; Pollack, L.M.; Bye, L.L. Disparities in smoking between the lesbian, gay, and bisexual population and the general population in California. Am. J. Public Health 2007, 97, 1496-1502. [CrossRef]

16. Deacon, R.M.; Mooney-Somers, J. Smoking prevalence among lesbian, bisexual and queer women in Sydney remains high: Analysis of trends and correlates. Drug Alcohol Rev. 2017, 36, 546-554. [CrossRef]

17. Schwan, R.; Allen, J.P. Unhealthy alcohol use. N. Engl. J. Med. 2005, 352, 596-607. 
18. Rehm, J.; Baliunas, D.; Borges, G.L.; Graham, K.; Irving, H.; Kehoe, T.; Parry, C.D.; Patra, J.; Popova, S.; Poznyak, V.; et al. The relation between different dimensions of alcohol consumption and burden of disease: An overview. Addiction 2010, 105, 817-843. [CrossRef]

19. Gonzales, G.; Przedworski, J.; Henning-Smith, C. Comparison of Health and Health Risk Factors Between Lesbian, Gay, and Bisexual Adults and Heterosexual Adults in the United States: Results from the National Health Interview Survey. Jama Intern. Med. 2016, 176, 1344-1351. [CrossRef]

20. Wong, C.F.; Kipke, M.D.; Weiss, G. Risk factors for alcohol use, frequent use, and binge drinking among young men who have sex with men. Addict. Behav 2008, 33, 1012-1020. [CrossRef]

21. Green, J.; Plant, M.A. Bad bars: A review of risk factors. J. Subst. Use 2007, 12, 157-189. [CrossRef]

22. Li, D.J.; Chen, S.L.; Yen, C.F. Multi-Dimensional Factors Associated with Illegal Substance Use Among Gay and Bisexual Men in Taiwan. Int. J. Env. Res. Public Health 2019, 16. [CrossRef] [PubMed]

23. Berman, A.H.; Palmstierna, T.; Kallmen, H.; Bergman, H. The self-report Drug Use Disorders Identification Test: Extended (DUDIT-E): Reliability, validity, and motivational index. J. Subst Abus. Treat. 2007, 32, 357-369. [CrossRef] [PubMed]

24. Hartz, S.M.; Oehlert, M.; Horton, A.C.; Grucza, R.A.; Fisher, S.L.; Culverhouse, R.C.; Nelson, K.G.; Sumerall, S.W.; Neal, P.C.; Regnier, P.; et al. Daily drinking is associated with increased mortality. Alcohol Clin. Exp. Res. 2018, 42, 2246-2255. [CrossRef] [PubMed]

25. Yen, C.F.; Kim, Y.S.; Tang, T.C.; Wu, Y.Y.; Cheng, C.P. Factor structure, reliability, and validity of the Chinese version of the School Bullying Experience Questionnaire. Kaohsiung J. Med. Sci. 2012, 28, 500-505. [CrossRef] [PubMed]

26. Yen, C.F.; Chou, W.J.; Liu, T.L.; Ko, C.H.; Yang, P.; Hu, H.F. Cyberbullying among male adolescents with attention-deficit/hyperactivity disorder: Prevalence, correlates, and association with poor mental health status. Res. Dev. Disabil. 2014, 35, 3543-3553. [CrossRef]

27. Chen, Y.C.; Hsu, C.C.; Hsu, S.H.; Lin, C.C. A Preliminary Study of Family Apgar Index. Acta. Paediatr. Sin. 1980, 21, 210-217.

28. Smilkstein, G. The family APGAR: A proposal for a family function test and its use by physicians. J. Fam. Pr. 1978, 6, 1231-1239.

29. Wang, C.C.; Lin, H.C.; Chen, M.H.; Ko, N.Y.; Chang, Y.P.; Lin, I.M.; Yen, C.F. Effects of traditional and cyber homophobic bullying in childhood on depression, anxiety, and physical pain in emerging adulthood and the moderating effects of social support among gay and bisexual men in Taiwan. Neuropsychiatr. Dis. Treat. 2018, 14, 1309-1317. [CrossRef]

30. Kelly, B.C.; Parsons, J.T. Prevalence and predictors of non-medical prescription drug use among men who have sex with men. Addict. Behav. 2010, 35, 312-317. [CrossRef]

31. Fallin, A.; Goodin, A.; Lee, Y.O.; Bennett, K. Smoking characteristics among lesbian, gay, and bisexual adults. Prev Med. 2015, 74, 123-130. [CrossRef] [PubMed]

32. Pollitt, A.M.; Mallory, A.B.; Fish, J.N. Homophobic Bullying and Sexual Minority Youth Alcohol Use: Do Sex and Race/Ethnicity Matter? Lgbt Health 2018, 5, 412-420. [CrossRef] [PubMed]

33. Tucker, J.S.; Ewing, B.A.; Espelage, D.L.; Green, H.D., Jr.; de la Haye, K.; Pollard, M.S. Longitudinal Associations of Homophobic Name-Calling Victimization with Psychological Distress and Alcohol Use During Adolescence. J. Adolesc. Health 2016, 59, 110-115. [CrossRef] [PubMed]

34. Purnell, J.Q.; Peppone, L.J.; Alcaraz, K.; McQueen, A.; Guido, J.J.; Carroll, J.K.; Shacham, E.; Morrow, G.R. Perceived discrimination, psychological distress, and current smoking status: Results from the Behavioral Risk Factor Surveillance System Reactions to Race module, 2004-2008. Am. J. Public Health 2012, 102, 844-851. [CrossRef]

35. Kosciw, J.G.; Palmer, N.A.; Kull, R.M. Reflecting resiliency: Openness about sexual orientation and/or gender identity and its relationship to well-being and educational outcomes for LGBT students. Am. J. Community Psychol. 2015, 55, 167-178. [CrossRef]

36. Dehue, F.; Bolman, C.; Vollink, T. Cyberbullying: youngsters' experiences and parental perception. Cyberpsychol. Behav. 2008, 11, 217-223. [CrossRef]

37. Vieno, A.; Gini, G.; Santinello, M. Different forms of bullying and their association to smoking and drinking behavior in Italian adolescents. J. Sch. Health 2011, 81, 393-399. [CrossRef]

38. Meyer, I.H. Minority stress and mental health in gay men. J. Health Soc. Behav. 1995, 36, 38-56. [CrossRef] 
39. Goldbach, J.T.; Schrager, S.M.; Dunlap, S.L.; Holloway, I.W. The application of minority stress theory to marijuana use among sexual minority adolescents. Subst. Use Misuse 2015, 50, 366-375. [CrossRef]

40. Peltzer, K. Prevalence and correlates of substance use among school children in six African countries. Int. J. Psychol. 2009, 44, 378-386. [CrossRef]

41. Matthews, A.K.; Steffen, A.; Hughes, T.; Aranda, F.; Martin, K. Demographic, Healthcare, and Contextual Factors Associated with Smoking Status Among Sexual Minority Women. Lgbt Health 2017, 4, 17-23. [CrossRef] [PubMed]

42. Assari, S.; Bazargan, M. Education Level and Cigarette Smoking: Diminished Returns of Lesbian, Gay and Bisexual Individuals. Behav. Sci. (Basel) 2019, 9. [CrossRef] [PubMed]

43. Ewing, B.A.; Osilla, K.C.; Pedersen, E.R.; Hunter, S.B.; Miles, J.N.; D'Amico, E.J. Longitudinal family effects on substance use among an at-risk adolescent sample. Addict. Behav. 2015, 41, 185-191. [CrossRef] [PubMed]

44. Ryan, C.; Huebner, D.; Diaz, R.M.; Sanchez, J. Family rejection as a predictor of negative health outcomes in white and Latino lesbian, gay, and bisexual young adults. Pediatrics 2009, 123, 346-352. [CrossRef]

45. Eisenberg, M.E.; Erickson, D.J.; Gower, A.L.; Kne, L.; Watson, R.J.; Corliss, H.L.; Saewyc, E.M. Supportive Community Resources Are Associated with Lower Risk of Substance Use among Lesbian, Gay, Bisexual, and Questioning Adolescents in Minnesota. J. Youth Adolesc. 2019. [CrossRef]

46. Pena, L.; Lorenzo-Blanco, E.I.; Perez, A.; Morello, P.; Arillo Santillan, E.; Kollath-Cattano, C.; Thrasher, J.F.; Sargent, J.; Mejia, R. Parental Style and Its Association with Substance Use in Argentinean Youth. Subst. Use Misuse 2017, 52, 518-526. [CrossRef]

47. Craig, S.L.; Austin, A.; Alessi, E. Gay affirmative cognitive behavioral therapy for sexual minority youth: A clinical adaptation. Clin. Soc. Work J. 2013, 41, 258-266. [CrossRef]

(C) 2020 by the authors. Licensee MDPI, Basel, Switzerland. This article is an open access article distributed under the terms and conditions of the Creative Commons Attribution (CC BY) license (http://creativecommons.org/licenses/by/4.0/). 